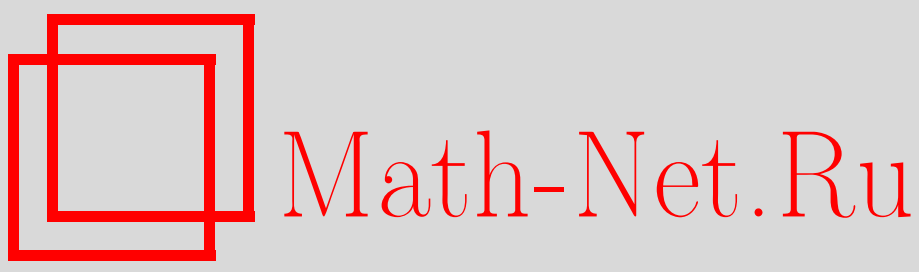

А. Ю. Воловиков, Об индексе $G$-пространств, Матем. сб., 2000, том 191, номер 9, 3-22

DOI: https://doi.org/10.4213/sm504

Использование Общероссийского математического портала Math-Net.Ru подразумевает, что вы прочитали и согласны с пользовательским соглашением

http: //www.mathnet.ru/rus/agreement

Параметры загрузки:

IP : 3.85 .5 .30

26 апреля 2023 г., $17: 55: 51$ 


\title{
Об индексе $G$-пространств
}

\begin{abstract}
С каждым $G$-пространством, где $G$ - компактная группа Ли, связан идеал кольца когомологий универсального классифицирующего пространства группы $G$, назьваемый идеалозначным индексом $G$-пространства.

Рассматривается фильтрация на идеалозначном индексе, естественньм образом возникающая из спектральной последовательности Лере. Исследуются свойства индекса с филштрацией, вводятся числовые индексы, удобные для получения оценок $G$-категории и изучения множества критических точек $G$-инвариантного функционала на многообразии.

Доказьвается обобщение теоремы Буржена-Янга для индекса с фильтрацией. Этот результат используется при нахождении оценки индекса множества частичных склеек для отображений пространства с действием $p$-тора в евклидово пространство.
\end{abstract}

Библиография: 29 названий.

\section{Введение}

По-видимому, впервые (ко)гомологический индекс в явном виде появился в работе Янга [1] для пространств со свободным действием групшы $\mathbb{Z}_{2}$. Этот индекс, тесно связанный с такими понятиями, как род, категория и когомологическая длина, нашел многочисленные применения в различных областях математики.

Для свободных действий произвольных конечных групп гомологические и геометрические индексы были введены Шварцем [2], [3], Коннером и Флойдом [4].

В дальнейшем, особенно в вопросах, связанных с нелинейньпи дифференциальными уравнениям, бифуркациями, критическими точками $G$-инвариантных функционалов на многообразиях и др., понадобилось рассматривать пространства с (не обязательно свободнњм) действием компактной группы Ли. Определению индексов таких $G$-пространств и их применениям были посвяшены многочисленные исследования, см., например, работу [5] и ссылки в ней.

До появления статьи Фаделла и Хуссейни [6] рассматриваются только числовые индексы: каждому $G$-пространству сопоставляется натуральное число (или $\infty)$ его индекс, причем так, что индекс прообраза не превосходит индекс образа при $G$-отображении. Это свойство индекса удобно использовать для доказательства того, что между конкретньми $G$-пространствами не сушествует $G$-отображения.

Результаты о несуществовании $G$-отображений часто приводят к важным следствиям.

Например, классическая теорема Борсука-Улама, утверждающая, что любое непрерывное отображение $n$-мерной сферы в $n$-мерное евклидово пространство

Работа вьполнена при финансовой поддержке Российского фонда фундаментальных исследований (грант № 97-01-00174) и фонда INTAS (грант № 96-0712).

(C) А.Ю. Воловиков 2000 
склеивает некоторую пару антиподальных точек на сфере, эквивалентна утверждению, что не существует $\mathbb{Z}_{2}$-отображения $S^{n} \rightarrow S^{n-1}$, где группа $\mathbb{Z}_{2}$ действует на сферах антиподальной инволюцией.

Таким образом, в данном случае факт несуществования эквивариантного отображения наоборот приводит к классической теореме существования.

С другой стороны, результаты подобного типа могут быть интересными сами по себе. Например, для того чтобы установить, что одно пространство не может быть вложено в другое, достаточно показать, что не существует эквивариантного отображения между их взрезанными квадратами, на которых действует естественная свободная инволюция, или более обшим образом рассмотреть другие конфигурационные пространства, связанные с заданными пространствами, на которых действуют подходящие группы. Результатом такого типа является, например, известная теорема ван Кампена-Флореса, утверждающая, что $k$-мерньй остов $(2 k+2)$-мерного симплекса нельзя вложить в $\mathbb{R}^{2 k}$.

Числовым индексом удобно пользоваться в случае действия групп, имеющих достаточно простое кольцо когомологий (типа алгебры многочленов от одной образуюшей). $\mathrm{K}$ таким группам, например, можно отнести циклическую группу простого порядка, окружность и группу кватернионов, по модулю равных единице. Для более сложных групп, например таких, как торы или $p$-торы, числовых инвариантов явно недостаточно. В связи с этим в работе [6] Фаделл и Хуссейни ввели так назьваемьй "идеалозначный" индекс - с каждым $G$-пространством $X$ связывается идеал $\operatorname{Ind}^{G} X$ кольца когомологий $\Lambda^{*}=H^{*}\left(B_{G}\right)$, где когомологии рассматриваются с коэффициентами в некотором поле $\mathbb{k}$ и $B_{G}-$ классифицирующее пространство групшы $G$.

Идеал $\operatorname{Ind}^{G} X$ определяется как ядро гомоморфизма эквивариантных когомологий, индуцированного отображением пространства $X$ в точку. Легко показать, см. [6], что если имеется $G$-отображение из $X$ в $Y$, то Ind ${ }^{G} Y \subset$ Ind $^{G} X$. Это позволяет во многих интересных случаях ответить на вопрос, существует $G$-отображение из $X$ в $Y$ или нет. С другой стороны, на идеалозначный индекс, по-видимому, нужно смотреть лишш как на первое препятствие к сушествованию эквивариантного отображения (возможньм подтверждением сказанного служит то, что идеалозначный индекс когомологической сферы есть главный идеал, порожденньй ее эквивариантным эйлеровьм классом).

В настоящей работе мы рассматриваем возрастающую фильтрацию на индекce Ind ${ }^{G} X$, естественным образом возникающую из спектральной последовательности Лере, и приводим применения, которые не могут быть получены только лишш с использованием индекса Ind ${ }^{G}$. В частности, в $\S 4$ мы приводим уточнение обошения теоремы Буржена-Янга, полученного в [6].

Числовые индексы, возникающие при нашем подходе, можно использовать практически так же, как индекс Янга [1] для группы $\mathbb{Z}_{2}$. В частности, имееют место результаты о частичных склейках орбит для пространств с действием $p$-тора, которые вполне аналогичны результатам для действия группы простого порядка, полученным в [7]. Эти утверждения приводятся в $\S 5$. Там же они применяются для получения вариантов теоремы ван Кампена-Флореса и цветной теоремы Тверберга.

В последнем параграфе изучается индекс множества критических точек $G$-инвариантного функционала и даются оценки $G$-категории. 


\section{§1. Фильтрация индекса}

Мы будем предполагать, что все пространства хаусдорфовы и наследственно паракомпактны, а отображения непрерьвны.

Когомологии, используюшиеся далее, - это когомологии Чеха с коэффициентами в поле $\mathbb{k}$, которое исключается из обозначений. Кроме того, для $p$-тора, т.е. элементарной абелевой $p$-группы $G=\mathbb{Z}_{p}^{n}=\mathbb{Z}_{p} \times \cdots \times \mathbb{Z}_{p}$, где $p$ - простое число, мы всегда будем считать, что $\mathbb{k}=\mathbb{Z}_{p}$ - поле из $p$ элементов, а для связной групшы Ли - что $\mathbb{k}=\mathbb{Q}$.

Для $G$-пространства $X$ положим $H_{G}^{*}(X)=H^{*}\left(E_{G} \times{ }_{G} X\right)$ (конструкция Бореля эквивариантных когомологий, см. [8; гл. 3]), где $E_{G} \rightarrow B_{G}$ - универсальное главное $G$-расслоение. Будем обозначать $H_{G}^{*}(\mathrm{pt})=H^{*}\left(B_{G}\right)$ через $\Lambda^{*}$, где pt одноточечное пространство.

Отображение $X \rightarrow$ pt индуцирует гомоморфизм $\Lambda^{*} \rightarrow H_{G}^{*}(X)$, ядро которого Фаделл и Хуссейни обозначают через $\operatorname{Ind}^{G} X$ и называют “идеалозначным" индексом $G$-пространства $X$, см. [6].

Из рассмотрения спектральной последовательности Лере расслоения $X_{G} \rightarrow B_{G}$ со слоем $X$ вытекает, что гомоморфизм $\Lambda^{*} \rightarrow H_{G}^{*}(X)$ разлагается в композицию

$$
\Lambda^{*} \rightarrow E_{2}^{*, 0} \rightarrow \cdots \rightarrow E_{r}^{*, 0} \rightarrow \cdots \rightarrow E_{\infty}^{*, 0} \subset H_{G}^{*}(X)
$$

и, следовательно, $\operatorname{Ind}^{G} X$ совпадает с ядром гомоморфизма $\Lambda^{*} \rightarrow E_{\infty}^{*, 0}$. Будем обозначать $\operatorname{Ind}^{G} X$ также через $\infty \operatorname{Ind}^{G} X$.

ОПРЕДЕЛЕНИЕ 1.1. Определим идеал $r \operatorname{Ind}^{G} X$ в $\Lambda^{*}$ как ядро гомоморфизма $H^{*}\left(B_{G}\right) \rightarrow E_{r+1}^{*, 0}$.

Из определения 1.1 следует, что

$$
{ }_{1} \text { Ind }^{G} X \subset{ }_{2} \text { Ind }^{G} X \subset \cdots \subset{ }_{k} \text { Ind }^{G} X \subset{ }_{k+1} \text { Ind }^{G} X \subset \cdots
$$

и объединение этих идеалов совпадает с $\operatorname{Ind}^{G} X=\infty \operatorname{Ind}^{G} X$.

ЗАмечание 1.2 . В случае, когда $X$ связно, $E_{r+1}^{*, 0}=H^{*}\left(B_{G}\right) / r \operatorname{Ind}^{G} X$.

Определим теперь соответствующие числовые индексы.

ОПРЕДЕЛЕНИЕ 1.3. Пусть $0 \neq \alpha \in \operatorname{Ind}^{G} X$. Определим $i_{X}(\alpha)$ как наименьшее $r$, для которого $\alpha \in{ }_{r} \operatorname{Ind}^{G} X$.

Минимум чисел $i_{X}(\alpha)$ по всем $0 \neq \alpha \in \operatorname{Ind}^{G} X$ обозначим через $i(X)$, а минимум по всем тем $\alpha \in \operatorname{Ind}^{G} X$, которые не являются делителями нуля в $\Lambda^{*}=H^{*}\left(B_{G}\right)$, обозначим через $i^{\prime}(X)$. В случае, когда необходимо явно указать действующую группу, будем обозначать индексы $i(X)$ и $i^{\prime}(X)$ через $i_{G}(X)$ и $i_{G}^{\prime}(X)$ соответственно.

Очевидно, что $i(X) \leqslant i^{\prime}(X)$.

Если $\operatorname{Ind}^{G} X=0$, то положим $i(X)=\infty$. Так, для пространств, имеющих непустое множество неподвижных точек, индекс $i(X)$ бесконечен. Аналогично, полагаем $i_{X}(\alpha)=\infty$, если класс $\alpha \in \Lambda^{*}$ не принадлежит $\operatorname{Ind}^{G} X$.

Отметим, что если $0 \neq \alpha \in$ Ind $^{G} X$, то в спектральной последовательности Лере расслоения $X_{G} \rightarrow B_{G}$ образ элемента $\alpha \in H^{*}\left(B_{G}\right)=\Lambda^{*}$ в $E_{2}^{*, 0}$ убивается некоторым дифференциалом. Номер этого дифференциала и есть $i_{X}(\alpha)$, а индекс $i(X)$ равен номеру первого ненулевого дифференциала, бьющего в нулевую строку. 
Можно определить фильтрацию для индекса и в категории “расслоений” (объекты- $G$-отображения $G$-пространств, морфизмы - соответствующие коммутативные квадраты $G$-отображений).

Пусть $X \rightarrow Y-G$-отображение $G$-пространств. Тогда оно индуцирует гомоморфизм $H_{G}^{*}(Y) \rightarrow H_{G}^{*}(X)$ эквивариантных когомологий, ядро которого обозначается через $\operatorname{Ind}_{Y}^{G} X$, см. [6]. Это ядро можно снабдить возрастающей фильтрацией, которая возникает из спектральной последовательности Лере отображения $X_{G} \rightarrow Y_{G}$. В случае $Y=\mathrm{pt}$ имеем $\operatorname{Ind}_{\mathrm{pt}}^{G} X=\operatorname{Ind}^{G} X$.

Таким образом, как и вьше, имеем

$$
\begin{aligned}
{ }_{1} \operatorname{Ind}_{Y}^{G} X & \subset{ }_{2} \operatorname{Ind}_{Y}^{G} X \subset \cdots \subset{ }_{k} \operatorname{Ind}_{Y}^{G} X \\
& \subset{ }_{k+1} \operatorname{Ind}_{Y}^{G} X \subset \cdots \subset \operatorname{Ind}_{Y}^{G} X=\operatorname{Ind}_{Y}^{G} X .
\end{aligned}
$$

\section{§2. Свойства индекса}

Следующее предложение вытекает непосредственно из определений.

ПРЕДЛОЖЕНИЕ 2.1. Пусть $X \rightarrow Y$ - эквивариантное отображсение $G$ пространств, тогда

1) ${ }_{k}$ Ind $^{G} Y \subset{ }_{k}$ Ind $^{G} X$, в частности Ind $^{G} Y \subset$ Ind $^{G} X$;

$2)$ in $X \leqslant$ in $Y, i(X) \leqslant i(Y) u i^{\prime}(X) \leqslant i^{\prime}(Y)$;

3) $i_{X}(\alpha) \leqslant i_{Y}(\alpha)$ для любого $0 \neq \alpha \in \operatorname{Ind}^{G} X$.

Числовой индекс in $X$ определен в [9], он равен наибольшему натуральному $d$ такому, что $\Lambda^{j} \rightarrow H_{G}^{j}(X)$ - мономорфизм для любого $j \leqslant d$ и $\Lambda^{d} \neq 0$.

В случае, когда $X$ - свободное $\mathbb{Z}_{2}$-пространство, индекс in $X$ совпадает с гомологическим индексом Янга [1].

Числовые индексы связаны между собой неравенствами

$$
\text { in } X \geqslant i(X)-1, \quad i^{\prime}(X) \geqslant i(X) .
$$

Кроме того, можно показать, используя лемму 1 из [10], что в случае действия группы $G=\mathbb{Z}_{2}$ справедливо соотношение in $X=i(X)-1$, а для окружности $\left(G=S^{1}\right)$ имеет место равенство in $X=i(X)-2$. Аналогичное утверждение справедливо и для группы $G=S^{3}$, а именно: в спектральной последовательности расслоения $X_{G} \rightarrow B_{G}$ при любом $r \geqslant 2$ модуль $E_{r}^{*, *}$ порождается над $\Lambda^{*}$ множеством $E_{r}^{0, *}$. Поскольку $\Lambda^{*}=H^{*}\left(B_{G}\right)=\mathbb{Q}[t]$, где $t$ имеет степень 4 , получаем, что in $X=i(X)-4$. Наконец, во всех трех указанных случаях $i(X)=i^{\prime}(X)$.

В случае действия группы $G=\mathbb{Z}_{p}$ простого порядка $p>2$ модуль $E_{r}^{*, *}$ порождается над $\Lambda^{*}$ объединением подмножеств $E_{r}^{0, *}$ и $E_{r}^{1, *}$ (см. [10; лемма 1]), поэтому $i(X)-1 \leqslant$ in $X \leqslant i(X)$.

ПРЕДЛОЖЕНИЕ 2.2. Предположим, что X представляется в виде обвединения двух замкнутых (или двух открытых) инвариантных подпространств $X=A \cup B$. Тогда

1) $\left({ }_{k} \operatorname{Ind}^{G} A\right) \cdot\left({ }_{m} \operatorname{Ind}^{G} B\right) \subset{ }_{k+m} \operatorname{Ind}^{G} X$,

2) $i^{\prime}(X) \leqslant i^{\prime}(A)+i^{\prime}(B)$

3) $i(X) \leqslant i(A)+i^{\prime}(B)$

4) $i(X) \leqslant i^{\prime}(A)+i(B)$ 
5) $i_{X}(\alpha \beta) \leqslant i_{A}(\alpha)+i_{B}(\beta)$, əде $\alpha \in \operatorname{Ind}^{G} A, \beta \in \operatorname{Ind}^{G} B u \alpha \beta \neq 0$.

Первые четыре утверждения вытекают из последнего утверждения, следующего непосредственно из леммы 2.7, доказываемой в конце $\S 2$.

Приведем теперь несколько следствий, касающихся действия на джойнах.

СлеДСТВИЕ 2.3. Пусть $X$ и $Y-G$-пространства, тогда

1) $\left({ }_{k} \operatorname{Ind}^{G} X\right) \cdot\left({ }_{m} \operatorname{Ind}^{G} Y\right) \subset{ }_{k+m} \operatorname{Ind}^{G}(X * Y)$,

2) $i^{\prime}(X * Y) \leqslant i^{\prime}(X)+i^{\prime}(Y)$,

3) $i(X * Y) \leqslant i(X)+i^{\prime}(Y)$

4) $i(X * Y) \leqslant i^{\prime}(X)+i(Y)$.

ДоказАТЕльство. $X$ и $Y$ естественньм образом вложены в $X * Y$. Применяя предложение $2.2 \mathrm{k} A=X * Y \backslash X$ и $B=X * Y \backslash Y$ и учитьвая, что эти подпространства эквивариантно стягиваются на $Y$ и $X$ соответственно, получаем требуемое.

Рассмотрим теперь частньй случай, когда в качестве $Y$ выступает $k$-мерная когомологическая сфера $S$ с сохраняющим ориентацию $G$-действием без неподвижных точек, т.е. такое пространство, что $H^{*}(S)=H^{*}\left(S^{k}\right)$, где $S^{k}$ - стандартная $k$-мерная сфера, и $G$ тривиально действует на $H^{k}(S)$. В этом случае $\operatorname{Ind}^{G} S=(e)$, где $e$ - эквивариантный класс Эйлера сферы $S$, т.е. эйлеров класс расслоения $S_{G} \rightarrow B_{G}$.

Обозначим через $m_{X}$ наименьшее натуральное $d>$ in $X$ такое, что $\Lambda^{d} \neq 0$.

СЛЕДСТВИЕ 2.4. Пусть $S$ является $k$-мерной когомологической сферой такой, что ее әквивариантный эйлеров класс не является делителем нуля в $\Lambda^{*}$. Тогда

$$
\begin{aligned}
\operatorname{in}(X * S) & <m_{X}+k+1, \\
i(X * S) & \leqslant i(X)+k+1, \\
i^{\prime}(X * S) & \leqslant i^{\prime}(X)+k+1 .
\end{aligned}
$$

ДокАЗАтЕльство. Из сделанных предположений следует, что $i^{\prime}(S)=k+1$, поэтому второе и третье неравенства вытекают из следствия 2.3.

Докажем первое неравенство. Из определения числа $m_{X}$ следует, что найдется класс $0 \neq \alpha \in \operatorname{Ind}^{G} X$ c $\operatorname{deg} \alpha=m_{X}$. Пусть $e-$ эквивариантный эйлеров класс когомологической сферы $S$. Тогда $\alpha e \neq 0$ и $\alpha e \in \operatorname{Ind}^{G} X * S$, следовательно, $\operatorname{in}(X * S)<\operatorname{deg} \alpha e=m_{X}+k+1$ (более того, in $(X * S)$ не превосходит наибольшего из натуральных чисел $d$ таких, что $d<m_{X}+k+1$ и $\Lambda^{d} \neq 0$ ), что и требовалось доказать.

Следствие 2.4 применимо в случае действия без неподвижных точек $p$-тора на компактной или паракомпактной и конечномерной когомологической сфере, поскольку в силу теоремы локализации Бореля, см. [8], эквивариантный эйлеров класс такой сферы не является делителем нуля в $\Lambda^{*}$. Отметим также, что в этом случае $m_{X}=$ in $X+1$.

Таким образом, в случае действия $G=\mathbb{Z}_{p}^{n}$ мы получаем следующие утверждения. 
А) Пусть $S$ либо компактно, либо паракомпактно и имеет конечную когомологическую размерность над полем $\mathbb{Z}_{p}$. Предположим, что $S$ является $k$-мерной когомологической сферой над $\mathbb{Z}_{p}$, m.e. $H^{*}(S) \cong H^{*}\left(S^{k}\right), u$ что $G=\mathbb{Z}_{p}^{n}$ действует на $S$ без неподвижних точек. Тогда in $S=k u$ $i(S)=i^{\prime}(S)=k+1$.

В) Пусть $S$ - когомологическая сфера из свойства А). Тогда in $(X * S) \leqslant$ in $X+$ in $S+1$, m.e. in $X \geqslant \operatorname{in}(X * S)-k-1$, где $X * S-$ джсойн пространств $X$ и $S$ с диагональным действием группь $G$. Аналогичные неравенства справедливы и для индексов $i$ и $i^{\prime}$, а именно $i(X * S) \leqslant i(X)+k+1 u i^{\prime}(X * S) \leqslant i^{\prime}(X)+k+1$. Отметим также, что $k$ нечетно, если $p$ нечетно (поскольку действие не имеет неподвижных точек). Этот факт хорошо известен в теории групп преобразований.

Аналогичные утверждения имеют место и в случае действия без неподвижных точек тора ( $k$-мерная когомологическая сфера $S$ либо компактна, либо является конечномерным паракомпактным пространством с конечным числом орбитных типов). Для действия тора имеем $m_{X}=$ in $X+2$. Неравенства для индексов $i$ и $i^{\prime}$ такие же, как в случае действия $p$-тора, а для индекса in неравенство имеет вид $\operatorname{in}(X * S) \leqslant$ in $X+k+2$. Кроме того, поскольку действие предполагается не имеющим неподвижных точек, $k$ - нечетное число.

Непосредственно из определений вытекает следующее предложение.

ПРЕДЛОЖЕНИЕ 2.5. Пусть $X$ и $Y-G$-пространства.

Eсли $\widetilde{H}^{i}(X)=0$ при $i<N$, mo $i(X) \geqslant N+1$.

Eсли $H^{i}(Y)=0$ при $i>N-1$ u $i(Y)<\infty$, mo $i(Y) \leqslant N$.

ЗАмЕчАниЕ 2.6. Из утверждения 2) предложения 2.1 следует, что для пространств $X$ и $Y$, удовлетворяющих условиям предложения 2.5, не существует $G$-отображения из $X$ в $Y$.

Предложение 2.2 вытекает из следуюшей леммы.

Лемма 2.7. Пусть $E=E_{1} \cup E_{2}$, где $E_{1}, E_{2}$ либо оба замкнутыл, либо оба открыты, $и \pi: E \rightarrow B-$ произвольное непрерывное отображсение. Пусть $\alpha_{1}, \alpha_{2} \in H^{*}(B)$ таковьи, что $\alpha_{i}$ убивается дифференииалом $d_{r_{i}}$ в спектральной последовательности Лере отображения $\left.\pi\right|_{E_{i}}: E_{i} \rightarrow B, i=1,2$. Тогда $\alpha_{1} \alpha_{2}$ убивается дифференциалом $d_{r} c r \leqslant r_{1}+r_{2}$ в спектральной последовательности Лере отображсения $E \rightarrow B$.

ДокАЗАТЕльство. Достаточно рассмотреть случай, когда оба подпространства $E_{1}, E_{2}$ открыты. Это следует из непрерывности когомологий и того факта, что в спектральных последовательностях можно переходить к прямому пределу.

Из построения (которое мы напоминаем ниже) спектральной последовательности Лере (см. [11], [12]) следует, что когомологии $H^{*}(E)$ являются когомологиями комплекса $C^{*}$ с убьвающей фильтрацией $F_{p} C^{*} \supset F_{p+1} C^{*}$. Аналогично $H^{*}\left(E_{i}\right)=H^{*}\left({ }_{i} C^{*}\right), i=1,2$, где ${ }_{i} C^{*}$ имеет фильтрацию $F_{p}\left({ }_{i} C^{*}\right)$. Гомоморфизм $H^{*}(E) \rightarrow H^{*}\left(E_{i}\right)$ индуцирован гомоморфизмом $C^{*} \rightarrow{ }_{i} C^{*}$, согласованным с фильтрациями, т.е. образ $F_{p} C^{*}$ при этом гомоморфизме содержится в $F_{p}\left({ }_{i} C^{*}\right)$. Более того, $C^{*} \rightarrow{ }_{i} C^{*}$, а также $F_{p} C^{*} \rightarrow F_{p}\left({ }_{i} C^{*}\right)$ являются эпиморфизмами.

Класс когомологий $\pi^{*} \alpha_{i}$ в $H^{*}(E)$ как класс, пришедший с базы, можно представить коциклом $c_{i} \in C^{p_{i}}, i=1,2$, где $c_{i} \in F_{p_{i}} C^{p_{i}}$ и $p_{i}=\operatorname{deg} c_{i}$. Обозначим образ $c_{i}$ в ${ }_{i} C^{p_{i}}$ через $c_{i}^{\prime}$. Тог да сушествует коцепь $a_{i} \in F_{p_{i}-r_{i}}\left({ }_{i} C^{p_{i}-1}\right)$ такая, что 
$c_{i}^{\prime}={ }_{i} d a_{i}$, где ${ }_{i} d-$ дифференциал в комплексе ${ }_{i} C^{*}$. Для $a_{i}$ можно найти коцепь $b_{i} \in F_{p_{i}-r_{i}} C^{p_{i}-1}$, образом которой $a_{i}$ является. Рассмотрим коцикл $c_{i}-d b_{i}$. Из того, что образ этой коцепи в ${ }_{i} C^{*}$ равен нулю, следует, что носитель коцепи $c_{i}-d b_{i}$ содержится в $E \backslash E_{i}$. Поэтому произведение $\left(c_{1}-d b_{1}\right)\left(c_{2}-d b_{2}\right)$ имеет пустой носитель и, следовательно, равно нулю. Таким образом,

$$
c_{1} c_{2}=c_{1} d b_{2}+d b_{1} \cdot c_{2}-d b_{1} \cdot d b_{2}=d\left( \pm c_{1} b_{2}+b_{1} c_{2}-b_{1} d b_{2}\right) .
$$

Коцепा $\pm c_{1} b_{2}+b_{1} c_{2}-b_{1} d b_{2}$ лежит по крайней мере в $F_{p_{1}+p_{2}-r_{1}-r_{2}} C^{*}$, откуда и следует требуемое утверждение.

Напомним теперь, как строится спектральная последовательность Лере (см. [11], [12]) отображения $\pi: E \rightarrow B$. Эта спектральная последовательность является спектральной последовательностью двойного комплекса

$$
K^{* *}=C^{*}\left(B ; \pi \mathscr{C}^{*}(E ; \mathbb{k})\right):=\Gamma_{B} \mathscr{C}^{*}\left(B ; \pi \mathscr{C}^{*}(E ; \mathbb{k})\right)=\mathscr{C}^{*}\left(B ; \pi \mathscr{C}^{*}(E ; \mathbb{k})\right)(B),
$$

здесь $\mathbb{k}$ - постоянный пучок на $E, \pi^{*}(E ; \mathbb{k})$ - прямой образ пучка $\mathscr{C}^{*}(E ; \mathbb{k}), \mathscr{C}^{*}-$ каноническая резольвента, $\Gamma_{B}$ - функтор сечения над $B$. Комплекс $C^{*}$, рассмотренньй вьше, определяется как $C^{n}=\sum_{p+q=n} K^{p q}$.

Аналогично, для отображения $\pi_{i}=\left.\pi\right|_{E_{i}}$ имеем ${ }_{i} C^{n}=\sum_{p+q=n}{ }_{i} K^{p q}$, где ${ }_{i} K^{* *}=C^{*}\left(B ; \pi_{i} \mathscr{C}^{*}\left(E_{i} ; \mathbb{k}\right)\right), i=1,2$.

Для открытого $U \subset B$ имеем

$$
\begin{aligned}
\pi \mathscr{C}^{*}(E ; \mathbb{k})(U) & =\mathscr{C}^{*}(E ; \mathbb{k})\left(\pi^{-1} U\right)=C^{*}\left(\pi^{-1} U ; \mathbb{k}\right) \\
\pi_{i} \mathscr{C}^{*}\left(E_{i} ; \mathbb{k}\right)(U) & =\mathscr{C}^{*}\left(E_{i} ; \mathbb{k}\right)\left(\pi_{i}^{-1} U\right)=C^{*}\left(E_{i} \cap \pi_{i}^{-1} U ; \mathbb{k}\right)=C^{*}\left(E_{i} \cap \pi^{-1} U ; \mathbb{k}\right)
\end{aligned}
$$

Из вялости канонической резольвенты следует, что

$$
C^{*}\left(\pi^{-1} U ; \mathbb{k}\right) \rightarrow C^{*}\left(E_{i} \cap \pi^{-1} U ; \mathbb{k}\right)
$$

- эпиморфизм, поэтому эпиморфизмом является гомоморфизм пучков $\pi^{\mathscr{C}} \mathscr{C}^{*}(E ; \mathbb{k}) \rightarrow$ $\pi_{i} \mathscr{C}^{*}\left(E_{i} ; \mathbb{k}\right)$. Из точности функтора $C^{*}(B ; \cdot)$ следует, что $K^{* *} \rightarrow{ }_{i} K^{* *}-$ эпиморфизм, $i=1,2$.

В заключение этого параграфа отметим свойство непрерывности индексов.

Пусть $X-G$-пространство и $A \subset X$ замкнуто и инвариантно. Тогда если $\alpha \in \operatorname{Ind}^{G} A$, то найдется инвариантная окрестность $N \supset A$ такая, что $i_{A}(\alpha)=i_{N}(\alpha)$.

Это следует из непрерывности когомологий и того, что в спектральных последовательностях можно переходить к прямому пределу (в силу точности последнего).

Поскольку в качестве пространства коэффициентов мы берем поле и $G$ - компактная группа Ли, $\Lambda^{j}$ при каждом $j$ является конечномерным векторньм пространством. Поэтому сушествует окрестность $N \supset A$ такая, что

$$
{ }_{k} \operatorname{Ind}^{G, j} A={ }_{k} \operatorname{Ind}^{G, j} N \quad \text { и } \quad i(A)=i(N), \quad i^{\prime}(A)=i^{\prime}(N), \quad \text { in } A=\text { in } N,
$$

где ${ }_{k} \operatorname{Ind}^{G, j} A=\Lambda^{j} \cap{ }_{k} \operatorname{Ind}^{G} A$ и аналогичным образом определяется ${ }_{k} \operatorname{Ind}^{G, j} N$. 


\section{§3. Некоторые применения}

В силу теоремы локализации Бореля, см. [8], если $Y$ компактно или паракомпактно и конечномерно и $G=\mathbb{Z}_{p}^{n}$ действует на $Y$ без неподвижных точек, то $i(Y)<\infty$. Аналогичное верно для компактного пространства с действием тора без неподвижных точек, а также для паракомпактного и конечномерного пространства с таким действием тора (без неподвижных точек), для которого число орбитных типов конечно.

Таким образом, из замечания 2.6 вытекает следующий результат, доказанный B [13].

ПРЕДЛОЖЕНИЕ 3.1. Пусть $G-p$-тор (m.e. $\left.G=\mathbb{Z}_{p}^{n}\right)$ или тор. Пусть $X$ и $Y$-G-пространства с действием без неподвижных точек, причем в случае действия тора $Y$ имеет конечное число орбитных типов. Предположсм, что $\widetilde{H}^{i}(X)=0$ при $i<N$ и что $Y$ компактно или паракомпактно и конечномерно и $H^{i}(Y)=0$ при $i>N-1$, где когомологии берутся с коэффициентами из поля $\mathbb{Z}_{p}$ в случае р-тора и из поля $\mathbb{Q}$ в случае тора. Тогда не существует G-отображения из $X$ в $Y$.

Часто в качестве $Y$ выступает $n$-мерная когомологическая сфера $S$ с сохраняющим ориентацию $G$-действием без неподвижных точек. В этом случае, как отмечалось выше, Ind ${ }^{G} S=(e)$, где $e$ - эквивариантный класс Эйлера сферы $S$, т.е. эйлеров класс расслоения $S_{G} \rightarrow B_{G}$ со слоем $S$.

Пусть $h: X \rightarrow S$ эквивариантно, тогда $e \in \operatorname{Ind}^{G} X$.

В этих предположениях нетрудно доказать справедливость следующего предложения.

ПРЕДЛОЖЕНИЕ 3.2. Предположсим, что е $\neq 0$ и е убивается трансгрессией в спектральной последовательности расслоения $X_{G} \rightarrow B_{G}$ (таким образом, $\left.i_{X}(e)=i_{S}(e)=\operatorname{deg} e\right)$. Тогда $h^{*} \neq 0$ в размерности $n=\operatorname{deg} e-1$.

Например, предложение 3.2 применимо в ситуации, когда $i(X)=\operatorname{deg} e$, поскольку из существования $G$-отображения $h: X \rightarrow S$ вытекает, что $i(X) \leqslant i_{X}(e) \leqslant$ $i_{S}(e)=\operatorname{deg} e$ и, следовательно, $i_{X}(e)=\operatorname{deg} e$. Таким образом, выполнены все условия предложения 3.2 , поэтому $0 \neq h^{*}: H^{n}(S) \rightarrow H^{n}(X)$, где $n=\operatorname{deg} e-1$. В частности, предложение 3.2 применимо для такого $X$, что $\widetilde{H}^{j}(X)=0$ при $j<n=$ $\operatorname{deg} e-1$ (действительно, из предложения 2.5 следует, что $i(X) \geqslant n+1=\operatorname{deg} e$ ). Последний факт является обобщением теоремы 1.6 из [14]. Отметим также, что в частном случае действия групшы $G=\mathbb{Z}_{p}^{n}$ предложение 3.2 применялось в доказательстве гипотезы Кнастера для $\mathbb{Z}_{p}^{n}$-симметричного множества точек на сфере и отображений в двумерную плоскость, см. доказательство теоремы 8 в [9] (а также доказательство теоремы 2 в [15]).

Следующее утверждение может рассматриваться как обратное к предложению 3.2. Его частньм случаем является теорема 3 работы [16].

ПРЕДЛОЖЕНИЕ 3.3. Пусть $h: X \rightarrow S$ - эквивариантное отображсение компактного $G$-пространства $X$ в $n$-мерную когомологическую сферу $S$, на которой $G$ действует без неподвижных точек. Предположим, как и выше, что әквивариантный эйлеров класс сферь $S$ отличен от нуля и что

1) $H^{n}(X) \cong \mathbb{k}$ и $G$ тривиально действует на $H^{n}(X)$, 
2) $H^{i}(X)=0$ npu $i>n$,

3) $h^{*} \neq 0$ в размерности $n$.

Тогда $i_{X}(e)=i_{S}(e)=\operatorname{deg} e=n+1 u \operatorname{Ind}^{G} X=\operatorname{Ind}^{G} S=(e)$.

ДокАЗАТЕльство. Из сделанных предположений следует, что $i_{X}(e) \leqslant n+1$. Пусть $e=d_{n+1} t$ в спектральной последовательности для $S$, где $t \in H^{n}(S)=\mathbb{k}$ - образующий. Положим $s=h^{*} t \in H^{n}(X)=\mathbb{k}=E_{2}^{0, n}$. Тогда $s$ - цикл всех дифференциалов, кроме, может быть, дифференциала $d_{n+1}$.

Обозначим $i(X)$ через $r_{0}$ и предположим, что $i(X)<n+1$. Без ограничения обшности можно считать, что $X$ связно, тогда в спектральной последовательности расслоения $X_{G} \rightarrow B_{G}$ со слоем $X$ имеем $E_{r}^{*, 0}=\Lambda^{*}$ при $2 \leqslant r \leqslant r_{0}$ и $E_{2}^{*, n}=E_{r}^{*, n}$ является модулем над $\Lambda^{*}$, изоморфным $\Lambda^{*}$ при $r \leqslant r_{0}$. Кроме того, найдется элемент $0 \neq \alpha \in \operatorname{Ind}^{G} X$ такой, что $i_{X}(\alpha)=r_{0}$ и, следовательно, $\alpha=d_{r_{0}} c$ для некоторого $c$.

Из соображений размерности следует, что $c s=0$, поэтому $0=d_{r_{0}}(c s)=\alpha s$. $\mathrm{C}$ другой стороны, $s$ является образующим модуля $E_{r_{0}}^{*, n}$ над $\Lambda^{*}$, изоморфного $\Lambda^{*}$, поэтому $\alpha s \neq 0$. Полученное противоречие показывает, что первый ненулевой дифференциал, бьющий в нулевую строку (в спектральной последовательности для пространства $X$ ), есть дифференциал $d_{n+1}$, причем так как $s=h^{*} t$, имеем $d_{n+1} s=e$. Теперь из свойства мультипликативности спектральной последовательности легко вытекает, что Ind ${ }^{G} X=\operatorname{Ind}^{G} S=(e)$.

Отметим, что требование тривиальности действия $G$ на $H^{n}(X)$ из условия 1$)$ автоматически выполнено для связной группы, а также для $p$-тора, поскольку в этом случае мы берем в качестве пространства коэффициентов поле из $p$ элементов.

Следуюшее утверждение непосредственно вытекает из предыдушего предложения и является обобщением основного результата работы [17].

СлеДСТВИЕ 3.4. Пусть $G=\mathbb{Z}_{p}^{n}$. Предположсим, что существует $G$-отображсние $f: M \rightarrow S$ связного замкнутого когомологического $N$-многообразия $M$ (ориентируемого в случае $p>2$ ) в $N$-мерную когомологическую сферу $S$ над $\mathbb{Z}_{p}$, на которой $G$ действует без неподвижних точек, такое, что $0 \neq f^{*}: H^{N}\left(S ; \mathbb{Z}_{p}\right) \rightarrow H^{N}\left(M ; \mathbb{Z}_{p}\right)$. Тогда если существует $G$-отображсение $M$ в $G$-пространство $K$ конечной когомологической размерности над полем $\mathbb{Z}_{p}$, то $0 \neq H^{j}\left(K ; \mathbb{Z}_{p}\right)$ для некоторого $j \geqslant N$. В частности, $\operatorname{dim}_{\mathbb{Z}_{p}} K \geqslant N$.

ДоказАтельство. Имеем $\operatorname{Ind}_{G} S=(e), \operatorname{deg} e=N+1$ и $i_{S}(e)=N+1$. Поскольку $f$ эквивариантно, из предложения 3.3 следует, что $i_{M}(e)=N+1$. Если бы $0=H^{j}\left(K ; \mathbb{Z}_{p}\right)$ для любого $j \geqslant N$, то $i(K) \leqslant N$ и мы получили бы противоречие с предположением о сушествовании $G$-отображения из $M$ в $K$.

Пусть $q=p^{n}$, где $p$ - простое нечетное число. Имеется свободное действие групшы $G=\mathbb{Z}_{p}^{n}$ на $\mathrm{SO}(q)$, см. [9].

Пусть заданы две непрерывные функции $f_{1}, f_{2}: \mathrm{SO}(q) \rightarrow \mathbb{R}$. Положим

$$
\begin{array}{r}
D\left(f_{1}, f_{2}\right)=\left\{x \in \mathrm{SO}(q): f_{1}(x)=f_{1}(g x) \forall g \in G \text { и } f_{2}(x)=f_{2}(g x)\right. \\
\text { для всех } g \in G, \text { кроме, возможно, одного элемента }\} .
\end{array}
$$

Мы покажем, что это множество непусто. Случай $p=3, n=1$ изучен в [18], а случай $p \geqslant 3, n=1-$ в [9]. 
Teорема 3.5. Множество $D\left(f_{1}, f_{2}\right)$ непусто.

ДокАЗАТЕльСТво. Поскольку $|G|=q$, на $Y^{q}$, где $Y$ - произвольное пространство, имеется естественное действие группы $G$ - действие на пространстве непрерывных отображений дискретной группы $G$ в $Y$.

Пусть $X-G$-пространство. По заданному отображению $f: X \rightarrow Y$ можно определить $G$-отображение $\widehat{f}: X \rightarrow Y^{q}$. Если точки пространства $Y^{q}$ формально записывать в виде $\sum y_{g} g$, то $\widehat{f}$ определяется формулой

$$
\widehat{f}(x)=\sum f\left(g^{-1} x\right) g
$$

(Приведенное построение $G$-отображения $\widehat{f}$ по $f$ годится для любой конечной группы $G$.)

Мы возьмем в качестве $X$ и $Y$ пространства $\mathrm{SO}(q)$ и $\mathbb{R}^{2}$ соответственно, а в качестве $f$ - отображение $f=\left(f_{1}, f_{2}\right): \mathrm{SO}(q) \rightarrow \mathbb{R}^{2}$, заданное формулой $f(x)=\left(f_{1}(x), f_{2}(x)\right), x \in \mathrm{SO}(q)$. Таким образом, получаем $G$-отображение $\widehat{f}: \operatorname{SO}(q) \rightarrow\left(\mathbb{R}^{2}\right)^{q}$.

Рассмотрим в $\left(\mathbb{R}^{2}\right)^{q}$ объединение $P=\Pi_{1} \cup \cdots \cup \Pi_{q}$ трехмерных плоскостей $\Pi_{i}$ вида

$$
\left\{\begin{array}{l}
x_{1}=\cdots=x_{q} \\
y_{1}=\cdots=y_{i-1}=\widehat{y}_{i}=y_{i+1}=\cdots=y_{q} \text { (координата } y_{i} \text { пропушена) }
\end{array}\right.
$$

где $(x, y)$ - координаты на плоскости $\mathbb{R}^{2}$.

Заметим, что $P$ инвариантно относительно $G$-действия, и поскольку диагональ $\Delta \subset\left(\mathbb{R}^{2}\right)^{q}$, состоящая из наборов вида $(v, \ldots, v), v \in \mathbb{R}^{2}$, содержится в $P$, группа $G$ действует на $\left(\mathbb{R}^{2}\right)^{q} \backslash P$ без неподвижных точек.

Кроме того, $D\left(f_{1}, f_{2}\right)=\widehat{f}^{-1}(P)$.

Нетрудно показать, что пространство $\left(\mathbb{R}^{2}\right)^{q} \backslash P$ эквивариантно гомотопически эквивалентно $(2 q-3)$-мерной сфере без конечного числа точек, т.е. букету $(2 q-4)-$ мерных сфер; приведенные когомологии такого пространства равны нулю во всех размерностях, кроме $2 q-4$, следовательно, $i\left(\left(\mathbb{R}^{2}\right)^{q} \backslash P\right)=2 q-3$.

$\mathrm{C}$ другой стороны, из [9] следует, что $i(\mathrm{SO}(q))=2 q-2$. Поэтому из предложения 3.1 получаем, что не сушествует эквивариантного отображения из $\mathrm{SO}(q)$ в $\left(\mathbb{R}^{2}\right)^{q} \backslash P$. Отсюда вытекает, что прообраз $P$ при отображении $\widehat{f}$ непуст, т.е. $D\left(f_{1}, f_{2}\right) \neq \varnothing$.

Из этой теоремы можно вывести следующий результат.

ТЕОРема 3.6. Пусть $R-\mathbb{Z}_{p}^{n}$-симметричное множество из q точек на сфере $S^{q-1}$ и $f_{1}, f_{2}: S^{q-1} \rightarrow \mathbb{R}-$ две непрерывные функиии. Тогда $R$ вращением можкно положить на поверхность уровня функиии $f_{1}$, причем так, что функиия $f_{2}$ будет принимать одно и то же значение на всех точках полученного множества, кроме, бъть мохет, одной.

Определение $\mathbb{Z}_{p}^{n}$-симметричного множества точек см. в [9]. 


\section{§4. Обобщения теорем типа Буржена-Янга}

Следующая теорема может рассматриваться как уточнение обобщения теоремы Буржена-Янга, полученного в [6].

Теорема 4.1. Пусть $X$ и $E-G$-пространства, $f: X \rightarrow E$ - эквивариантное отображсние и $P \subset E$ - замкнутое инвариантное подпространство. Положим $A=f^{-1} P$. Тогда

$$
\left({ }_{k} \operatorname{Ind}^{G}(E \backslash P)\right) \cdot\left({ }_{m} \operatorname{Ind}^{G} A\right) \subset{ }_{k+m} \operatorname{Ind}^{G} X .
$$

В частности, $i^{\prime}(A) \geqslant i^{\prime}(X)-i^{\prime}(E \backslash P)$.

ДоказАтельство. Обозначим $E \backslash P$ через $B$. Пусть $i_{A}(\alpha)=m$ и $i_{B}(\beta)=k$, где $\alpha \in{ }_{m} \operatorname{Ind}^{G} A, \beta \in{ }_{k} \operatorname{Ind}^{G} B$ и $\alpha \beta \neq 0$. Из непрерывности когомологий следует, что найдется открытая инвариантная окрестность $U$ замкнутого множества $A$ такая, что $i_{U}(\alpha)=m$. Поскольку $U \cup B=X$, из утверждения 5) предложения 2.2 вытекает, что $i_{X}(\alpha \beta) \leqslant m+k$. Это доказывает первое утверждение теоремы. Второе утверждение является прямым следствием первого.

СлеДСТВИЕ 4.2. Пусть $X$ и $E-G$-пространства, $f: X \rightarrow E-$ эквивариантное отображсение, $P \subset E$ - замкнутое инвариантное подпространство. Предположим, что для некоторого $0 \neq \alpha \in \operatorname{Ind}^{G}(E \backslash P)$ имеет место неравенство $i_{X}(\alpha)>i_{E \backslash P}(\alpha)$.

Тогда $f^{-1} P$ непусто.

В частности, $f^{-1} P \neq \varnothing$, если выполнено одно из неравенств:

a) $i(X)>i(E \backslash P)$,

b) $i^{\prime}(X)>i^{\prime}(E \backslash P)$,

c) in $X>\operatorname{in}(E \backslash P)$.

ДокАЗАТЕЛЬСтво. Предположим противное. Тогда имеется $G$-отображение $X \rightarrow E \backslash P$. Воспользовавшись свойством 2) индексов из предложения 2.1 , приходим к противоречию.

Теорема 3.5 и результаты $\S 5$ являются конкретными приложениями следствия 4.2.

В связи с доказанным следствием упомянем еще один факт (см. [9; предложение 6]): если имеется класс $0 \neq \beta \in H_{G}^{j}(E)$ такой, что образ $\beta$ в $H_{G}^{j}(E \backslash P)$ тривиален, но образ $\beta$ в $H_{G}^{j}(E)$ при гомоморфизме $f_{G}^{*}: H_{G}^{j}(E) \rightarrow H_{G}^{j}(X)$, индуцированном отображением $f$, отличен от нуля, то $f^{-1} P$ непусто. Если при этом $X$ является $l$-мерным когомологическим многообразием над $\mathbb{k}$, то $\operatorname{dim}_{\mathbb{k}} f^{-1} P \geqslant \max \left(\operatorname{dim} X^{G}, l-j\right)$, где $X^{G}$ - множество неподвижных точек действия $G$ на $X$ и через $\operatorname{dim}_{\mathbb{k}}$ обозначается когомологическая размерность над полем $\mathbb{k}$. В частности, если $X$ является $l$-мерным когомологическим многообразием над $\mathbb{k}$ и имеется такой класс $0 \neq \alpha \in \operatorname{Ind}^{G}(E \backslash P)$, что $\alpha \notin \operatorname{Ind}^{G} X$ (т.е. $\left.i_{X}(\alpha)=\infty>i_{E \backslash P}(\alpha)\right)$, то $\operatorname{dim}_{\mathbb{k}} f^{-1} P \geqslant l-\operatorname{deg} \alpha$.

Теорема 4.1 переносится также и в категорию расслоений, а именно рассмотрим следующую коммутативную диаграмму, в которой $P \subset E$ замкнуто:

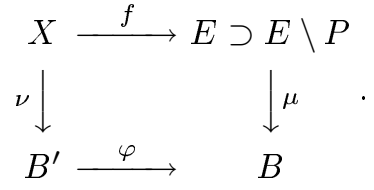


Коммутативность диаграммы означает, что $\mu f x=\varphi \nu x$ для любого $x \in f^{-1}(E \backslash P)$.

Предположим далее, что все пространства являются $G$-пространствами, $P$ инвариантно относительно $G$-действия и все отображения в диаграмме эквивариантны.

Tеорема 4.3. Положим $A=f^{-1} P$. Тогда

$$
\left(\varphi_{G}^{*}\left({ }_{k} \operatorname{Ind}_{B}^{G}(E \backslash P)\right)\right) \cdot\left({ }_{m} \operatorname{Ind}_{B^{\prime}}^{G} A\right) \subset{ }_{k+m} \operatorname{Ind}_{B^{\prime}}^{G} X .
$$

В ситуации, когда $k, m=\infty$, этот результат использовался в [19] при обобщении теоремы Дольда-Накаока [20], [21].

\section{§5. Частичные склейки}

Пусть $X-G$-пространство, где $G$ - конечная группа, и $f: X \rightarrow Y$ - отображение в пространство $Y$.

Определим подмножество $A(f)$ как множество точек, на орбитах которых $f$ постоянно (можно сказать, что $f$ склеивает орбиты точек из $A(f)$ ). Таким образом,

$$
A(f)=\{x \in X: f(x)=f(g x) \forall g \in G\} .
$$

Множество $A(f)$ замкнуто и инвариантно. Как было показано в начале доказательства теоремы 3.5 , имеется $G$-отображение $\widehat{f}: X \rightarrow Y^{|G|}$. При этом $A(f)=$ $f^{-1}(\Delta)$, где $\Delta$ - диагональ в $Y^{|G|}$, совпадающая с множеством неподвижных точек действия $G$ на $Y^{|G|}$.

В случае, когда $X$ - свободное $\mathbb{Z}_{2}$-пространство и $Y-m$-мерное евклидово пространство, оценка индекса множества полных склеек $A(f)$ отображения $f: X \rightarrow$ $\mathbb{R}^{m}$ дается теоремой Буржена-Янга [1], утверждающей, что in $A(f) \geqslant$ in $X-m$.

Это неравенство прямо вытекает из теоремы 4.1, поскольку для свободного действия групшы $\mathbb{Z}_{2}$ имеем $i^{\prime}(X)=$ in $X+1, i^{\prime}(A(f))=$ in $A(f)+1$ и $i^{\prime}\left(\left(\mathbb{R}^{m}\right)^{2} \backslash \Delta\right)=m$.

Классическая теорема Борсука-Улама является непосредственным следствием теоремы Буржена-Янга, поскольку для $n$-мерной сферы $S^{n}$ со свободным $\mathbb{Z}_{2}$-действием имеем in $S^{n}=n$.

Пусть подмножество $P \subset Y^{|G|}$ замкнуто и инвариантно и $P \subset \Delta$. При условии, что последнее включение является строгим, множество $\widehat{f}^{-1}(P)$ естественно назвать множеством частичных склеек, поскольку $f$ для некоторых точек из $\widehat{f}^{-1}(P)$ склеивает только часть орбиты. С помошью теоремы 4.1 или следствия 4.2 можно попытаться установить, что множество $\widehat{f}^{-1}(P)$ непусто. Однако для этого нужно знать не только индекс пространства $X$, но и индекс пространства $Y^{|G|} \backslash P$, найти который даже в простейших случаях достаточно сложно (если $Y=\mathbb{R}^{m}$ и $P$ объединение плоскостей, то $Y^{|G|} \backslash P$ - конфигурационное пространство). С другой стороны, нужна лишш хорошая оценка сверху этого индекса. Согласно предложению 2.5 оценку сверху можно найти, зная размерность, начиная с которой все группы когомологий пространства $Y^{|G|} \backslash P$ равны нулю.

Основываясь на этих соображениях, мы рассмотрим один конкретный случай.

Далее в этом параграфе $G$ - $p$-тор, т.е. $G=\mathbb{Z}_{p}^{n}, p$ - простое число, и $q=$ $p^{n}=|G|$. 
Пусть $X-G$-пространство. Для $f: X \rightarrow Y$ и $2 \leqslant k \leqslant q$ положим

$A(f, k)=\left\{x \in X:\right.$ существуют $k$ различных элементов $g_{1}, \ldots, g_{k} \in G$ таких, что $\left.f\left(g_{1} x\right)=\cdots=f\left(g_{k} x\right)\right\}$.

Заметим, что $A(f, q) \subset A(f, q-1) \subset \cdots \subset A(f, 2)$ и $A(f, q)$ совпадает с $A(f)$.

Пусть $k$ фиксировано, $2 \leqslant k \leqslant q$. Определим замкнутое подмножество $P_{k} \subset Y^{q}$ как множество точек $\left(y_{1}, \ldots, y_{q}\right) \in Y^{q}$ таких, что $y_{i_{1}}=\cdots=y_{i_{k}}$ для некоторых $1 \leqslant i_{1}<\cdots<i_{k} \leqslant q$.

Заметим, что $P_{k}$ инвариантно относительно $G$-действия на $Y^{q}$ и $A(f, k)=$ $\widehat{f}^{-1}\left(P_{k}\right)$, где $G$-отображение $\widehat{f}: X \rightarrow Y^{q}$ определено в начале доказательства теоремы 3.5 .

Как уже отмечалось выше, нахождение точного значения индекса $i\left(Y^{q} \backslash P_{k}\right)$, по-видимому, является достаточно сложной задачей. Поэтому далее мы оценим сверху этот индекс в случае $Y=\mathbb{R}^{m}$ с помощью результата Коэна-Ласка [7].

Итак, пусть $Y=\mathbb{R}^{m}$.

Точку в $\left(\mathbb{R}^{m}\right)^{q}$ будем задавать $q$-набором $\left(v_{1}, \ldots, v_{q}\right)$, где $v_{j} \in \mathbb{R}^{m}$. Тогда $P_{k}$ - объединение всех плоскостей в $\left(\mathbb{R}^{m}\right)^{q}$ вида $v_{i_{1}}=\cdots=v_{i_{k}}, i_{1}<\cdots<i_{k}$. Как и вьше, отображение $f: X \rightarrow \mathbb{R}^{m}$ определяет эквивариантное отображение $\widehat{f}: X \rightarrow\left(\mathbb{R}^{m}\right)^{q}$ и $A(f, k)=\widehat{f}^{-1}\left(P_{k}\right)$.

В [7] показано, что если $k \geqslant(q+1) / 2$ или $k=2$, то

$$
H^{j}\left(\left(\mathbb{R}^{m}\right)^{q} \backslash P_{k}\right)=0 \text { при } j>(m-1)(q-1)+k-2 .
$$

Поскольку $G$ действует на $\left(\mathbb{R}^{m}\right)^{q} \backslash P_{k}$ без неподвижных точек, из предложения 2.5 следует, что

$$
i^{\prime}\left(\left(\mathbb{R}^{m}\right)^{q} \backslash P_{k}\right)<(m-1)(q-1)+k
$$

Отсюда и из следствия 4.2 вытекает

ТеОРема 5.1. Пусть $f: X \rightarrow \mathbb{R}^{m}$ - непрерывное отображсение $G$-пространства $X$ в $\mathbb{R}^{m}$. Предположим, что $i(X) \geqslant(m-1)(q-1)+k u k \geqslant(q+1) / 2$ или $k=2$. Тогда $A(f, k) \neq \varnothing$.

ЗАмЕчАнИЕ 5.2. Эта теорема остается справедливой, если заменить индекс $i(X)$ на $i^{\prime}(X)$. Более того, в этом случае из теоремы 4.1 получаем, что $i^{\prime}(A(f, k)) \geqslant$ $i^{\prime}(X)-(m-1)(q-1)-k+1$.

СледСтвиЕ 5.3. Пусть $f: X \rightarrow \mathbb{R}^{m}$. Если $\widetilde{H}^{i}(X)=0$ nрu $i<(m-1) \times$ $(q-1)+k-1 u k \geqslant(q+1) / 2$ uлu $k=2$, mo $A(f, k) \neq \varnothing$.

Это следствие можно получить прямым объединением результатов Клэпп-Пуппе [13] (см. следствие 3.1 вьше) и Коэна-Ласка [7]. Однако требование ацикличности слишком ограничительно, и в некоторых случаях приходится пользоваться именно теоремой 5.1. В качестве примера мы рассмотрим случай отображения в евклидово пространство симплициального взрезанного произведения джойна остовов симплексов, представляющий интерес в связи с теоремами типа ван КампенаФлореса и Тверберга.

Обозначим через $\Delta^{N}$ симплекс размерности $N$ и через $\Delta_{s}^{N}$ его $s$-мерный остов. 
Следуя [22], [23], назовем набор из $q$ подмножеств некоторого множества $j$-дизъюнктным, где $2 \leqslant j \leqslant q$, если пересечение любых $j$ подмножеств из этого $q$-набора пусто.

Пусть $K-$ симплициальный комплекс и $2 \leqslant j \leqslant q$. Определим $P_{j}^{q}(K) \subset K^{q}$ как объединение таких произведений $\sigma_{1} \times \cdots \times \sigma_{q}$ симплексов из $K$, что $q$-набор симплексов $\sigma_{1}, \ldots, \sigma_{q} \subset K$ является $j$-дизъюнктным.

Симметрическая группа $\Sigma_{q}$ действует на $K^{q}$ и на его подпространстве $P_{j}^{q}(K)$ перестановками координат. Поскольку $|G|=q$, можно вложить $G$ в $\Sigma_{q}$ (для этого упорядочим элементы $G$ и рассмотрим $G$ как $G$-множество, тогда каждый элемент определит подстановку).

Обозначим через $J^{q}(X)$ джойн $q$ экземпляров пространства $X$. Его можно реализовать как факторпространство $X^{q} \times \Delta^{q-1} / \sim$ по отношению эквивалентности:

$$
\left(x_{1}, \ldots, x_{q}, t_{1}, \ldots, t_{q}\right) \sim\left(x_{1}^{\prime}, \ldots, x_{q}^{\prime}, t_{1}^{\prime}, \ldots, t_{q}^{\prime}\right),
$$

если $t_{i}=t_{i}^{\prime}$ при всех $i$ и $x_{i}=x_{i}^{\prime}$ для тех $i$, для которых $0 \neq t_{i}=t_{i}^{\prime}$ (здесь $x_{i}, x_{i}^{\prime} \in X$ и точки симплекса $\Delta^{q-1}$ представляются векторами $\left(t_{1}, \ldots, t_{q}\right) \in \mathbb{R}^{q}$ с $t_{i} \geqslant 0$ и $\left.\sum t_{i}=1\right)$. Образ точки $(z, \theta)$, где $z=\left(x_{1}, \ldots, x_{q}\right) \in X^{q}$ и $\theta=\left(t_{1}, \ldots, t_{q}\right) \in \Delta^{q-1}$, в $J^{q}(X)$ обозначим через $[z, \theta]$.

Группа $\Sigma_{q}$ действует на $X^{q}, \Delta^{q-1}$ и на $J^{q}(X)$ перестановками координат.

Для симплициального комплекса $K$ при $2 \leqslant j \leqslant q$ можно определить два подпространства $\widetilde{J}_{j}^{q}(K) \subset J_{j}^{q}(K)$ в $J^{q}(K)$, инвариантных относительно действия $\Sigma_{q}$. Подпространство $\widetilde{J}_{j}^{q}(K) \subset J_{j}^{q}(K)$ определяется как объединение симплексов вида $\sigma_{1} * \cdots * \sigma_{q}$, где $\sigma_{1}, \ldots, \sigma_{q} \subset K-j$-дизъюнктный $q$-набор симплексов. Если считать, что джойн пространства с пустьм множеством совпадает с самим пространством, и рассмотреть объединение симплексов указанного выше вида, но допуская наличие пустых симплексов в $q$-наборе, то получится пространство $J_{j}^{q}(K)$, содержашее $\widetilde{J}_{j}^{q}(K)$. Таким образом, $J_{j}^{q}(K)$ получается из $\widetilde{J}_{j}^{q}(K)$ добавлением некоторых “граничных" точек пространства $J^{q}(K)$ (граничная точка имеет вид $[z, \theta]$, где $\left.\theta \in \partial \Delta^{q-1}=\Delta_{q-2}^{q-1}\right)$.

Легко видеть, что $G$ действует на $P_{j}^{q}(K)$ и на $J_{j}^{q}(K)$ без неподвижных точек. Если $K$ - конечномерный комплекс, то оба пространства $P_{j}^{q}(K)$ и $J_{j}^{q}(K)$ являются конечномерными клеточньми комплексами и, следовательно, их индексы $i(\cdot), i^{\prime}(\cdot)$ и in $(\cdot)$ конечны.

Чтобы можно было воспользоваться теоремой 5.1, нужно иметь нижние оценки индексов $i(\cdot)$ или $i^{\prime}(\cdot)$.

B [24] была найдена нижняя оценка индекса in ( · ) этих пространств в случае, когда $K$ - джойн комплексов вида $\Delta_{s-1}^{N}$; там показано, что если для некоторых натуральных $k_{i}$ при $i=1, \ldots, l$ вьполнены неравенства $\left(k_{i}+1\right)(q-1)+q s_{i} \geqslant$ $\left(N_{i}+1\right)(j-1)$ и $K=\Delta_{s_{1}-1}^{N_{1}} * \cdots * \Delta_{s_{l}-1}^{N_{l}}$, то

$$
\begin{aligned}
& \text { in } J_{j}^{q}(K) \geqslant\left(N_{1}+\cdots+N_{l}+l\right)(j-1)-\left(k_{1}+\cdots+k_{l}+l\right)(q-1)-1, \\
& \text { in } P_{j}^{q}(K) \geqslant\left(N_{1}+\cdots+N_{l}+l\right)(j-1)-\left(k_{1}+\cdots+k_{l}+l+1\right)(q-1)-1 .
\end{aligned}
$$

Для индексов $i(\cdot)$ и $i^{\prime}(\cdot)$ аналогичная оценка получается тем же методом с использованием предложения 2.1 и свойств индексов $A, B$ из $\S 2$. Оказывается, что правые части неравенств больше на единицу. Таким образом, имеет место следующий результат. 
ПРЕДЛОЖЕНИЕ 5.4. Пусть для некоторых натуральных $k_{i}$ nри $i=1, \ldots, l$ выполнены неравенства $\left(k_{i}+1\right)(q-1)+q s_{i} \geqslant\left(N_{i}+1\right)(j-1)$. Положим $K=\Delta_{s_{1}-1}^{N_{1}} * \cdots * \Delta_{s_{l}-1}^{N_{l}} \cdot$ Тогда

$$
\begin{aligned}
& i\left(J_{j}^{q}(K)\right) \geqslant\left(N_{1}+\cdots+N_{l}+l\right)(j-1)-\left(k_{1}+\cdots+k_{l}+l\right)(q-1), \\
& i\left(P_{j}^{q}(K)\right) \geqslant\left(N_{1}+\cdots+N_{l}+l\right)(j-1)-\left(k_{1}+\cdots+k_{l}+l+1\right)(q-1) .
\end{aligned}
$$

Это предложение дает и нижнюю оценку для индекса $i^{\prime}(\cdot)$, поскольку $i^{\prime}(\cdot) \geqslant i(\cdot)$.

ТЕОРЕМА 5.5. Пусть для некоторых натуральных $k_{i}$ при $i=1, \ldots, l$ выполнены неравенства $\left(k_{i}+1\right)(q-1)+q s_{i} \geqslant\left(N_{i}+1\right)(j-1)$. Предположсим, что задано непрерывное отобрахсение $f: K \rightarrow \mathbb{R}^{m}$, где $K=\Delta_{s_{1}-1}^{N_{1}} * \cdots * \Delta_{s_{l}-1}^{N_{l}}$, u пусть $k \geqslant(q+1) / 2$.

Тогда если $\left(N_{1}+\cdots+N_{l}+l\right)(j-1) \geqslant\left(k_{1}+\cdots+k_{l}+l+m\right)(q-1)+k$, то найдется $j$-дизбюнктный $k$-набор симплексов $\sigma_{1}, \ldots, \sigma_{k}$ из $K$ такой, что $f\left(\sigma_{1}\right) \cap \cdots \cap f\left(\sigma_{k}\right) \neq \varnothing$.

ДокАЗАТЕЛьство. Проекция произведения $K^{q}$ на первый сомножитель определяет отображение $h: P_{j}^{q}(K) \rightarrow \mathbb{R}^{m}$. Утверждение теоремы эквивалентно тому, что множество $A(h, k)$ непусто. Последнее вытекает из предположенного в теореме неравенства, теоремы 5.1 и оценки индекса $i\left(P_{j}^{q}(K)\right)$ из предложения 5.4.

В случае $k=q$ более общее утверждение (для отображений в многообразие) доказано в [24] с помощью оценки индекса in $P_{j}^{q}(K)$, приведенной перед предложением 5.4. Переформулировка этого утверждения при $j=2$ (см. [25; теорема 1]), получающаяся, если взять $k_{1}=\cdots=k_{l}=0$, может быть, в частности, интерпретирована как вариант цветной теоремы Тверберга, см. [25]. Случай $k<q$ рассмотрен в [26] для $l=1$ и в [25] для произвольного $l$ в ситуации, когда $q-$ простое число, и тоже в качестве следствия приводит к варианту цветной теоремы Тверберга, см. [25].

Положим $k_{1}=\cdots=k_{l}=0$ и заменим неравенства $\left(k_{i}+1\right)(q-1)+q s_{i} \geqslant$ $\left(N_{i}+1\right)(j-1), i=1, \ldots, l$, равенствами:

$$
\left(N_{i}+1\right)(j-1)=q\left(s_{i}+1\right)-\alpha_{i}-1, \quad \text { где } \alpha_{i} \geqslant 0, \quad i=1, \ldots, l .
$$

Поскольку $N_{i} \geqslant s_{i}-1$, имеем

$$
0 \leqslant \alpha_{i} \leqslant(q-j+1) s_{i}+q-1 \text {. }
$$

Кроме того, число $q\left(s_{i}+1\right)-\alpha_{i}-1$ должно делиться на $j-1$.

Прямое вычисление показывает, что неравенство из теоремы 5.5 можно переписать в виде

$$
q\left(s_{1}+\cdots+s_{l}\right) \geqslant \alpha_{1}+\cdots+\alpha_{l}+m(q-1)+k .
$$

Таким образом, из теоремы 5.5 получаем следующий результат, в котором $k \geqslant 2$ и через $\nu(k)$ обозначается наименьшее из всех натуральных чисел, не меньших чем $k$ и являющихся степенью простого числа (легко показать, не используя постулат Бертрана, что $\nu(k) \leqslant 2 k-2$, см. ниже). 
СлеДСТвИЕ 5.6. Пусть $\left(N_{i}+1\right)(j-1)=\nu(k)\left(s_{i}+1\right)-\alpha_{i}-1$, әде $0 \leqslant \alpha_{i} \leqslant$ $(\nu(k)-j+1) s_{i}+\nu(k)-1$, и число $\nu(k)\left(s_{i}+1\right)-\alpha_{i}-1$ делится на $j-1, i=1, \ldots, l$. Предположим, что задано непрерьвное отображение $f: K \rightarrow \mathbb{R}^{m}$, где $K=$ $\Delta_{s_{1}-1}^{N_{1}} * \cdots * \Delta_{s_{l}-1}^{N_{l}}$.

Тогда если $\nu(k)\left(s_{1}+\cdots+s_{l}\right) \geqslant \alpha_{1}+\cdots+\alpha_{l}+m(\nu(k)-1)+k$, то найдется $j$-дизбюнктный $k$-набор симплексов $\sigma_{1}, \ldots, \sigma_{k}$ из $K$ такой, что $f\left(\sigma_{1}\right) \cap \cdots \cap$ $f\left(\sigma_{k}\right) \neq \varnothing$.

ДокАЗАТЕЛьство. Покажем, что $k>(\nu(k)+1) / 2$. Действительно, это очевидно, если $k$ - степень простого числа, поскольку тогда $k=\nu(k)$. В противном случае для некоторого $d$ имеем $2^{d}<k<2^{d+1}$ и из определения числа $\nu(k)$ следует, что $\nu(k) \leqslant 2^{d+1}$. Поэтому $(\nu(k)+1) / 2 \leqslant\left(2^{d+1}+1\right) / 2=2^{d}+\frac{1}{2}<k$. Теперь результат вытекает из теоремы 5.5 .

Такими же как в [25] рассуждениями, используя следствие 5.6 при $j=2$, получаем еще один вариант цветной теоремы Тверберга, в которой для подмножества $X \subset \mathbb{R}^{m}$ через conv $X$ обозначается его выпуклая оболочка и в случае, когда точки $X$ покрашены в $l$ цветов, через $\chi_{i}(X)$ обозначается число точек в $X$, покрашенных в $i$-й цвет.

СлеДСТВИЕ 5.7. Положим $N_{i}=\nu(k)\left(s_{i}+1\right)-\alpha_{i}-2$, әде $0 \leqslant \alpha_{i} \leqslant\left(s_{i}+1\right) \times$ $(\nu(k)+1), i=1, \ldots, l$. Предположим, что в $\mathbb{R}^{m}$ задано конечное подмножсество $X$, состоящее из $l+\sum_{i=1}^{l} N_{i}$ точек, покрашенных в $l$ иветов, причем так, что ровно $N_{i}+1$ точек покрашены в $i$-й ивет, $i=1, \ldots, l$.

Тогда если $\nu(k)\left(s_{1}+\cdots+s_{l}\right) \geqslant \alpha_{1}+\cdots+\alpha_{l}+m(\nu(k)-1)+k$, то в $X$ найдутся $k$ подмнохсеств $M_{1}, \ldots, M_{k}$ таких, что

1) $M_{j} \cap M_{j^{\prime}}=\varnothing$ npu $j \neq j^{\prime}$,

2) $\chi_{i}\left(M_{j}\right) \leqslant s_{i}$ при всех $i=1, \ldots, l$ и $j=1, \ldots, k$,

3) $\operatorname{conv} M_{1} \cap \cdots \cap \operatorname{conv} M_{k} \neq \varnothing$.

ДокАЗАТЕЛЬСтво. Определим по линейности непрерывное отображение $\Delta^{N_{1}} * \cdots * \Delta^{N_{l}} \rightarrow \mathbb{R}^{m}$, при котором вершины симплекса $\Delta^{N_{i}}$ переходят в $N_{i}+1$ точек $i$-го цвета, и обозначим через $f$ его ограничение на $K=\Delta_{s_{1}-1}^{N_{1}} * \cdots * \Delta_{s_{l}-1}^{N_{l}}$. Тогда из следствия 5.6 (при $j=2$ ) получаем, что найдутся попарно непересекающиеся симплексы $\sigma_{1}, \ldots, \sigma_{k} \subset K$ такие, что $f\left(\sigma_{1}\right) \cap \cdots \cap f\left(\sigma_{k}\right) \neq \varnothing$. Это означает, что conv $M_{1} \cap \cdots \cap \operatorname{conv} M_{k} \neq \varnothing$, где $M_{j}$ состоит из образов вершин симплекса $\sigma_{j}$, $j=1, \ldots, k$. Ясно также, что $M_{j} \cap M_{j^{\prime}}=\varnothing$ при $j \neq j^{\prime}$. Наконец, поскольку $\sigma_{j} \subset K$, в образе вершин симплекса $\sigma_{j}$ при отображении $f$ не более $s_{i}$ вершин $i$-го цвета при каждом $i=1, \ldots, l$.

\section{§ 6. Индекс множества критических точек $G$-инвариантного функционала}

Свойства индексов из предложений 2.1 и 2.2 могут быть использованы при построении теории Люстерника-Шнирельмана для инвариантного функционала на многообразии с действием компактной группы Ли $G$.

Для краткости рассмотрим случай гладкого действия группы Ли $G$ на гладком компактном конечномерном многообразии $M$. Пусть $f: M \rightarrow \mathbb{R}$ - гладкая $G$-инвариантная функция на $M$. Орбита критической точки целиком состоит из критических точек. Поэтому множество критических точек является $G$-пространством. 
Классический минимаксньй метод Люстерника-Шнирельмана оценки числа критических точек через категорию многообразия (см., например, [27]) можно перенести почти дословно в эквивариантную ситуацию, заменяя категорию на подходящий индекс [5], [13], [26], [28]. Сформулируем соответствуюший результат для индекса $i^{\prime}$, обладаюшего всеми необходимыми свойствами.

Обозначим через $M_{j}$ семейство всех компактных $G$-инвариантных подмножеств $X \subset M$ таких, что $i^{\prime}(X) \geqslant j$. Положим

$$
\lambda_{j}=\inf _{X \in M_{j}} \sup _{x \in X} f(x) .
$$

Теорема 6.1. Предположим, что $i^{\prime}(M)<\infty$. Тогда на любой поверхности уровня $f^{-1}\left(\lambda_{j}\right)$ имеется хотя бъ одна критическая орбита.

Eсли $\lambda_{j}=\lambda_{j+1}=\cdots=\lambda_{j+k}$, mо $i^{\prime}\left(S \cap f^{-1}\left(\lambda_{j}\right)\right) \geqslant k+1$, где $S$-множество критических точек функиии $f$.

При доказательстве используются свойство 2) из предложения 2.2 и непрерывность индекса: у всякого компактного $G$-инвариантного подмножества $X \subset M$ имеется компактная $G$-инвариантная окрестность $N$ такая, что $i^{\prime}(X)=i^{\prime}(N)($ см. конец $\S 3)$.

В [6] идеалозначный индекс использовался для развития метода Люстерника-Шнирельмана: с каждьм идеалом кольца $\Lambda^{*}$, содержащим $\operatorname{Ind}^{G} M$, связывалось критическое значение функционала. Эти результаты допускают введение индекса фильтрации. Для краткости определим лишп соответствующие критические значения, снова ограничившись случаем компактного конечномерного многообразия.

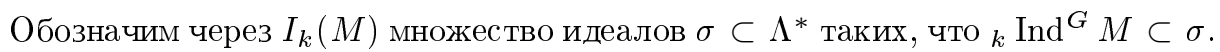
Для $\sigma \in I_{k}(M)$ через $M_{\sigma, k}$ обозначим семейство всех компактных $G$-инвариантных подмножеств $X \subset M$ таких, что ${ }_{k}$ Ind $^{G} X \subset \sigma$.

Положим

$$
\lambda_{\sigma, k}=\inf _{X \in M_{\sigma, k}} \sup _{x \in X} f(x) .
$$

Тогда поверхность уровня $\left\{f=\lambda_{\sigma, k}\right\}$ содержит хотя бы одну критическую орбиту.

Число критических орбит оценивается снизу через $G$-категорию многообразия, см., например, [13], [29]. В свою очередь, $G$-категорию можно оценить снизу через индекс. Пусть $X \subset M$ - замкнутое $G$-инвариантное подмножество в $M$ ( $M$ не обязательно многообразие). Тогда $G$-саt $M X$ - это наименьшее $k$ такое, что $X$ представляется в виде объединения $k$ замкнутых инвариантных подмножеств $X_{1}, \ldots, X_{k}$ и вложение $X_{i} \subset M$ при любом $i$ эквивариантно гомотопно $G$-отображению пространства $X_{i}$ в некоторую орбиту $G / G_{x}$, где $x \in M$ и $G_{x}-$ стационарная подгруппа точки $x$. При $X=M$ будем обозначать $G$-саt $X X$ просто через $G$-cat $X$. Отметим также, что каждое $G$-отображение $X_{i}$ в соответствующую орбиту можно продолжить до $G$-отображения в эту же орбиту некоторой открытой инвариантной окрестности множества $X_{i}$ в $X$ (см., например, [13]).

Предположим, что $i^{\prime}(M)<\infty$. Тогда $i^{\prime}\left(G / G_{x}\right)<\infty$ для орбиты любой точки $x \in M$. Пусть $G$-саt $M X=k$. Тогда согласно предложению 2.4 из [13] имеется $G$-отображение $X$ в джойн $k$ (не обязательно различных) орбит $X \rightarrow G / G_{x_{1}} * \cdots *$ $G / G_{x_{k}}$, где $x_{1}, \ldots, x_{k} \in M$. Поэтому

$i^{\prime}(X) \leqslant i^{\prime}\left(G / G_{x_{1}} * \cdots * G / G_{x_{k}}\right) \leqslant i^{\prime}\left(G / G_{x_{1}}\right)+\cdots+i^{\prime}\left(G / G_{x_{k}}\right) \leqslant k \cdot \max _{1 \leqslant i \leqslant k} i^{\prime}\left(G / G_{x_{i}}\right)$. 
Положим $d_{M}=\max _{x \in M} i^{\prime}\left(G / G_{x}\right)$ и $d_{X}=\max _{x \in X} i^{\prime}\left(G / G_{x}\right)$. Тогда $G$-cat $M X$ $i^{\prime}(X) / d_{M}$ и $G$-cat $X \geqslant i^{\prime}(X) / d_{X}$. (Например, если $G$ - тор, то условие $i^{\prime}(M)<\infty$ эквивалентно отсутствию неподвижных точек действия и $i^{\prime}\left(G / G_{x}\right)=2$. Поэтому $G$-cat $M \geqslant i^{\prime}(X) / 2$.)

В частности, $i^{\prime}\left(G / G_{x}\right) \leqslant \operatorname{dim} G / G_{x}+1 \leqslant \operatorname{dim} G+1$ для любой точки $x \in M$. Поэтому

$$
G-\operatorname{cat}_{M} X \geqslant \frac{i^{\prime}(X)}{d(M)} \geqslant \frac{i^{\prime}(X)}{\operatorname{dim} G+1},
$$

где $d(M)=\max _{x \in M} \operatorname{dim} G / G_{x}=\operatorname{dim} G-\min _{x \in M} \operatorname{dim} G_{x}$, а также $G$-cat $X \geqslant$ $i^{\prime}(X) / d(X)$, где $d(X)=\max _{x \in X} \operatorname{dim} G / G_{x}=\operatorname{dim} G-\min _{x \in X} \operatorname{dim} G_{x}$.

Наконец, отметим, что если при сделанных выше предположениях группа $G$ конечна, то $\operatorname{dim}\left(G / G_{x_{1}} * \cdots * G / G_{x_{k}}\right)=k-1$. Поэтому $i^{\prime}\left(G / G_{x_{1}} * \cdots * G / G_{x_{k}}\right) \leqslant k$ и, следовательно, $G$-саt $M X i^{\prime}(X)$.

Пусть $H$ - замкнутая подгруппа в $G$. Поскольку каждое $G$-пространство $X$ является также и $H$-пространством, определен индекс $i_{H}^{\prime}(X)$ (отвечающий групе $H$ и подходящим коэффициентам в когомологиях); $G$-отображение $X \rightarrow G / G_{x_{1}} * \cdots *$ $G / G_{x_{k}}$, где $x_{1}, \ldots, x_{k} \in M$ и $k=G$-cat $_{M} X$, является также $H$-отображением, поэтому из свойств индекса вытекает неравенство

$$
i_{H}^{\prime}(X) \leqslant G-\operatorname{cat}_{M} X \cdot \max _{1 \leqslant j \leqslant k} i_{H}^{\prime}\left(G / G_{x_{j}}\right)
$$

Если $i_{H}^{\prime}(M)<\infty$, то $\max _{1 \leqslant j \leqslant k} i_{H}^{\prime}\left(G / G_{x_{j}}\right)$ - конечное число, не превосходящее $d_{M, H}=\max _{x \in M} i_{H}^{\prime}\left(G / G_{x}\right)$. Поэтому $G$-cat ${ }_{M} X \geqslant i_{H}^{\prime}(X) / d_{M, H}$. Кроме того, $d_{M, H} \leqslant d(M)+1$ (разумеется, это неравенство имеет место лишь при условии, что индексы всех орбит конечны). Таким образом, мы получили следующий результат.

Теорема 6.2. Предположичм, что $i_{H}^{\prime}(M)<\infty$. Тогда

$$
G-\operatorname{cat}_{M} X \geqslant \frac{i_{H}^{\prime}(X)}{d_{M, H}} \geqslant \frac{i_{H}^{\prime}(X)}{d(M)+1} \geqslant \frac{i_{H}^{\prime}(X)}{\operatorname{dim} G+1} .
$$

Если в этой теореме взять $M=X$, то получаем, что при $i_{H}^{\prime}(X)<\infty$ имеют место неравенства

$$
G \text { - cat } X \geqslant \frac{i_{H}^{\prime}(X)}{d_{X, H}} \geqslant \frac{i_{H}^{\prime}(X)}{d(X)+1} \geqslant \frac{i_{H}^{\prime}(X)}{\operatorname{dim} G+1},
$$

где $d_{X, H}=\max _{x \in X} i_{H}^{\prime}\left(G / G_{x}\right)$.

СлЕДСТВИЕ 6.3. Пусть $\widetilde{H}^{j}\left(X ; \mathbb{Z}_{p}\right)=0$ nри $j<N$, әде $p$ - некоторое простое число. Предположсим, что в $G$ имеется подгруппа $H$, являющаяся р-тором и действующая без неподвижсных точек на $M$. Тогда

$$
G-\operatorname{cat}_{M} X \geqslant \frac{N+1}{d_{M, H}} \geqslant \frac{N+1}{d(M)+1} \geqslant \frac{N+1}{\operatorname{dim} G+1} .
$$


ДокАЗАТЕльство. Из предложения 2.5 следует, что $i_{H}^{\prime}(X) \geqslant N+1$, и результат вытекает из теоремы 6.2 , поскольку из того, что $p$-тор $H$ действует на любой орбите $G$-действия $G / G_{x}$ без неподвижных точек, следует, что $i_{H}^{\prime}\left(G / G_{x}\right)<\infty$ и $d_{M, H}<\infty$.

СЛЕДСТВИЕ 6.4. Пусть $X$ - такое $G$-пространство, что $\widetilde{H}^{j}\left(X ; \mathbb{Z}_{p}\right)=0$ при $j<N$, где $p$ - некоторое простое число. Предполохим, что в $G$ имеется конечная подгруппа $H$, действующая свободно на $X$, порядок которой делится на простое число р. Тогда

$$
G-\operatorname{cat}_{M} X \geqslant \frac{N+1}{d(M)+1} \quad u \quad G \text {-cat } X \geqslant \frac{N+1}{d(X)+1} .
$$

ДокАЗАТЕльство. В $H$ имеется циклическая подгруппа простого порядка $p$ (т.е. $p$-тор ранга 1 ), действуюшая свободно на $M$, поэтому применимо следствие 6.3 .

ЗАмечАниЕ 6.5. Оценки $G$-категории для ацикличных (до некоторой размерности $N)$ пространств получены в [13], [14], [29].

Автор благодарен Е. Г. Скляренко за многочисленные полезные обсуждения.

Работа над настоящей статьей была начата в сентябре 1997 года во время пребывания автора в Университете Glasgow Caledonian в группе нелинейных систем и хаоса отделения математики, сотрудникам которого автор приносит искреннюю благодарность за гостеприимство.

\section{Список литературы}

1. Yang C. T. On theorems of Borsuk-Ulam, Kakutani-Yamabe-Yujobo and Dyson. I // Ann. of Math. 1954. V. 60. № 2. P. 262-282.

2. Швари A. C. Некоторые оценки рода топологического пространства в смысле Красносельского // УМН. 1957. Т. 12. № 4. С. 209-214.

3. Швари A. С. Род расслоенного пространства // Труды ММО. 1962. Т. 11. С. 99-126.

4. Conner P.E., Floyd E.E. Fixed point free involutions and equivariant maps // Bull. Amer. Math. Soc. (N. S.). 1960. V. 66. P. 416-441.

5. Fadell E., Husseini S. Relative cohomological index theories // Adv. Math. 1987. V. 64. P. 1-31.

6. Fadell E., Husseini S. An ideal-valued cohomological index theory with applications to Borsuk-Ulam and Bourgin-Yang theorems // Ergodic theory and dynamic systems. 1988. V. 8. Spec. Issue. P. 259-268.

7. Cohen F., Lusk E. L. Configuration-like spaces and the Borsuk-Ulam theorem // Proc. Amer. Math. Soc. 1976. V. 56. P. 313-317.

8. Hsiang W. Y. Cohomology theory of topological transformation groups. Berlin: SpringerVerlag, 1975.

9. Воловиков А. Ю. Теорема типа Буржена-Янга для $\mathbb{Z}_{p}^{n}$-действия // Матем. сб. 1992 . T. 183. № 7. C. 115-144.

10. Chang T., Skjelbred T. Group actions on Poincaré duality spaces // Bull. Amer. Math. Soc. (N. S.). 1972. V. 78. P. 1024-1026.

11. Bredon G. Sheaf theory. New York: McGraw-Hill, 1988.

12. Godement $R$. Topologie algébrique et théorie des faisceaux. Paris: Hermann, 1958.

13. Clapp M., Puppe D. Critical point theory with symmetries // J. Reine Angew. Math. 1991. V. 418. P. 1-29.

14. Marzantowicz $W$. Borsuk-Ulam theorem for any compact Lie group // J. London Math. Soc. (2). 1994. V. 49. № 2. P. 195-208. 
15. Воловиков А. Ю. Об отображениях многообразий Штифеля со свободньм $\mathbb{Z}_{p}^{n}$-действием в многообразия // УМН. 1992. Т. 47. №6. С. 27-56.

16. Звягин В.Г. Вычисление коиндекса эквивариантного фредгольмова отображения // Труды НИИ математики ВГУ. Воронеж. 1974. Т. 15. С. 36-43.

17. Daccach J. A. Nonexistence of equivariant degree one maps // Proc. Amer. Math. Soc. 1992. V. 101. № 3. P. 530-532.

18. Макеев В. В. Задача Кнастера и почти сферические сечения // Матем. сб. 1989. Т. 180. №3. C. 424-431.

19. Воловиков А. Ю. О послойных G-отображениях // УМН. 1996. Т. 51. № 3. С. 189-190.

20. Dold A. Parametrized Borsuk-Ulam theorems // Comment. Math. Helv. 1988. V. 63. P. 275-285.

21. Nakaoka M. Parametrized Borsuk-Ulam theorems and characteristic polinomials // Lecture Notes in Math. 1989. V. 1411. P. 155-170.

22. Sarkaria K. S. A generalized van Kampen-Flores theorem // Proc. Amer. Math. Soc. 1991. V. 111. № 2. P. 559-565.

23. Sarkaria K. S. A generalized Kneser conjecture // J. Combin. Theory. Ser. B. 1990. V. 49. P. 236-240.

24. Воловиков А.Ю. К теореме ван Кампена-Флореса // Матем. заметки. 1996. Т. 59. № 5. C. $663-670$.

25. Богатьй C. А. Цветная теорема Тверберга // Вестник МГУ. Сер. 1. Матем., мех. 1999. № 3. C. $14-19$.

26. Богатый С. А. Геометрия отображений в евклидово пространство // УМН. 1998. Т. 53. № 5. C. $27-56$.

27. Дубровин Б.А., Новиков С.П., Фоменко А.Т. Современная геометрия. Методы теории гомологий. М.: Наука, 1984.

28. Fadell $E$. The equivariant Ljusternik-Schnirelmann method for invariant functionals and relative cohomological index theories // Métodes topologiques en analyse non linéare. Sémin. Math. Super., Semin. Sci. OTAN (NATO Adv. Study Inst.), Montreal. 1985. V. 95. P. 41-70.

29. Marzantowicz $W$. A G-Lusternik-Schnirelman category of space with an action of a compact Lie group // Topology. 1989. V. 28. № 4. P. 403-412. 